\title{
Automatic PC disassembly for component recovery
}

\begin{abstract}
In this article, a personal computer disassembly cell is presented. With this cell, a certain degree of automatism is afforded for the non-destructive disassembly process and for the recycling of these kinds of mass-produced electronic products. Each component of the product can be separated. The disassembly cell is composed of several sub-systems, each of which is dedicated to the planning and execution of one type of task. A computer vision system is employed for the recognition and localisation of the product and of each of its components. The disassembly system proposed here also has a modelling system for the products and each of its components, the information necessary for the planning of tasks, generating the disassembly sequence and planning of the disassembly movements. These systems co-operate with each other to achieve a semi-automatic disassembly of the product.
\end{abstract}

Keywords Non-destructive disassembly $\cdot$ Automatic disassembly $\cdot$ Recognition and location of components . Component recovery

\section{Introduction}

In the past, technological innovations arose from the need to survive. Nowadays, technological changes also come about from the desire to improve the quality of life. However, natural resources of raw materials for industrial use are limited. During the industrial revolution, no consideration was given to our natural

F. Torres $(\bowtie) \cdot$ P. Gil $\cdot$ S. T. Puente $\cdot$ J. Pomares

Dept. of Physics, Systems Engineering and Signal Theory, University of Alicante, Crta. San Vicente s/n.,

PO Box 99, Alicante, Spain

E-mail: fernando.torres@ua.es

Tel.: + 34-965-903682

Fax: + 34-965-909750

R. Aracil

DISAM, Polytechnic University of Madrid, Madrid, Spain surroundings when designing new products. Today, ecology is increasingly being considered at the design stage of new products because of the great amount of industrial residues that are generated [1]. Many governments impose laws concerning the recycling of products, to take better advantage of components and/ or raw materials for recycling. In 1998, the European Commission introduced a draft law (Waste Electrical and Electronic Equipment) concerning the management of electrical and electronic residues and which was most recently amended in April 2002.

The key to efficient recycling is in the disassembly of the product, allowing the removal of material, components and/or toxic substances.

The phases of the service life of a product [2] can be divided into:

1. The design of the product: Where the product is made according to what the desired model is and the information available about the different components that it will be composed of, considering its environmental impact and its ease of recycling and reusability.

2. The manufacture of the product: Where the product is made according to its original design.

3. The use of the product: This phase may vary according to the individual user.

4. The recovery of the product: The last phase, where the different parts are recycled for their re-use in the building of other new products.

The recycling is aimed at recovering the materials made available by disassembling and separating them through chemical processes.

Disassembly is the key process in the recovery of the re-usable parts of a product, as well as in the separation of its materials. This is an important process, not only in the recycling of products, but also in their systematic maintenance where a damaged or defective component is replaced by another.

Two different types of disassembly may be considered, either destructive or non-destructive, depending on 
the kind of operation that has to be made: automatic disassembly techniques are used in maintenance work to avoid high-risk manual tasks, and the degree of such disassembly depends on the type of components involved and/or the type of work to be done. The process does not conclude with a disassembly, it continues later with an assembly that allows the recovery of the operability of the product (i.e. car assembly plant, changing brake shoes, oil changes, wheels, battery, etc.). In such cases the disassembly tasks serve as a guide for a subsequent assembly, and this has to be done by means of a non-destructive process.

When a disassembly is done to recycle a product by separating its components and/or raw materials, the use, or not, of destructive techniques in a given operation also depends on the sorting relationship between the materials and components, defined by the manufacturing process used for the product.

The disassembly process of a product can be total or partial. For example, in a maintenance application, only the components to be replaced and those that prevent their dismounting have to be disassembled, whereas in a recycling application, the disassembly of the entire product is generally necessary.

\section{System architecture}

An automatic disassembly process is justified when a flexible manufacturing cell is able to carry out a continuous disassembly operation. This contrasts with what happens in an assembly process where the manufacture of a given type of product for a certain period of time is well-known and based on the demand for the product. The disassembly process is different because the same type of product is seldom introduced into the process one after another, so the disassembly process is influenced by a great variety of models, and even different types of products, which therefore creates great uncertainty regarding not only their order but their structural configuration [3].

A flexible manufacturing cell is therefore recommended for disassembly processes in which a great capacity of recognition and adaptability are required, due to the constant appearance of different types of products. In addition, this should be as automatic as possible and achieved within the shortest possible time. Thus, a certain rhythm can be guaranteed in the process, and its automation will be justified.

In this study, the research has concentrated on partial or total non-destructive disassembly techniques. The architecture of the system presented here is shown in Fig. 1. The global planner receives the order to disassemble a product either totally or partially. The component to be disassembled must be specified for the process to begin.

Once the product in the knowledge base has been identified from the information captured and processed by the computer vision system, the product modelling is

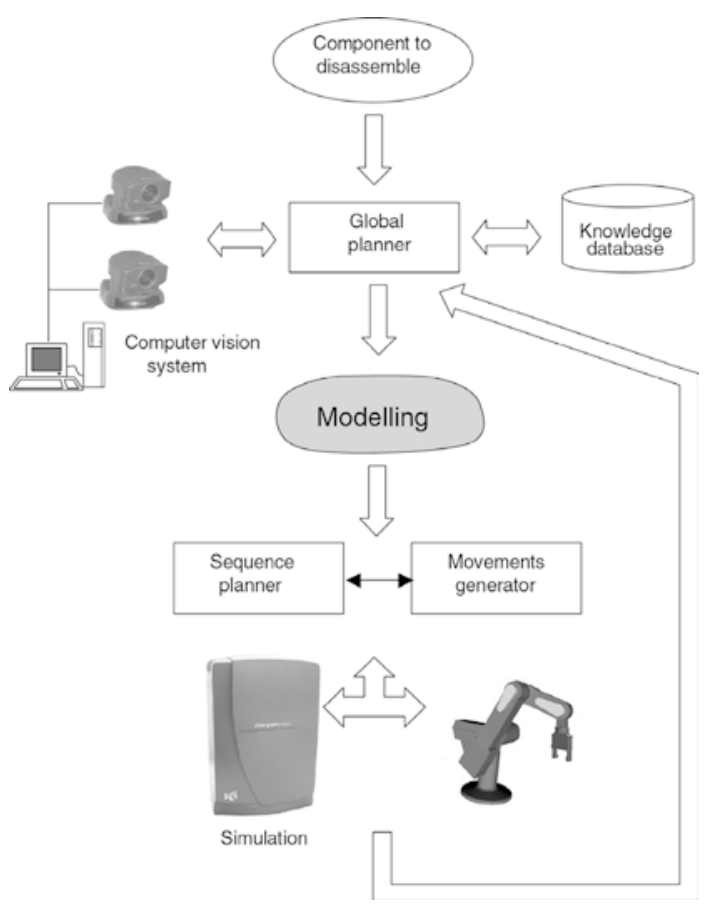

Fig. 1 Architecture of the automatic disassembly process

generated. This is completed gradually from the disassembly process and from the information stored in the knowledge base, captured and processed by the computer vision system. Initially, a generic relational model (generic graph of hierarchic relations among the different components that make up the product) is extended gradually with the process. This generic relational model is the base of the "sequence planner" module. A geometric product model is additionally generated as a grouping of components from the relational model that exists at that moment, the components stored in the knowledge base, and from the location information of the components obtained through the computer vision system. The geometric model obtained is used by the "movements generator" module (Fig. 1).

From the existing relational model, the aim of the sequence planner is to automatically select the components that are to be removed in order to disassemble another one, and to establish the order in which each of them must be separated - all of which must take place without any further operations being necessary.

The aim of the movements generator module is to generate the Cartesian trajectory for separating a component from the rest of the assembly. The movements that the robot must make to disassemble a given component, considering the rest of the assembly and the kinematics of the robot to be used as constraints, must be determined. Once the sequence of the movements has been generated and before the real automatic disassembly is carried out by the robotic system, there is a simulation module that allows the visualisation of the disassembly process (Fig. 1).

The remaining sections of this article describe how each of the modules are integrated in the automatic 
disassembly process (Fig. 1). Finally, a real example of a PC non-destructive partial disassembly is presented.

\subsection{Computer vision system}

The sensory part of the disassembly process is formed by the computer vision system. The two aims of this exercise are the recognition and location of the components and fasteners as well as the product itself.

\subsubsection{Architecture employed}

The acquisition system consists of a pair of SONY EVID31 cameras in a stereoscopic system. These motorized cameras are located at the end the $y-z$ Cartesian robot, which allows the pair of cameras to be positioned perpendicular to the base of the robot and the worktable (Fig. 2). A greater degree of freedom is therefore achieved, which permits the capture of the images from more angles, and facilitates not only the recognition but also the location process. The worktable has been marked to facilitate the location process. The acquisition and processing systems are completed with a minicamera mounted at the end of the robot, and a MatroxGenesis image acquisition and processing card. In Fig. 3, the recognition and location scheme is shown.

\subsubsection{Recognition}

The recognition process has two basic objectives: on the one hand, to determine which element is the product and which are its components, and on the other, to detect the components that are to be disassembled. This is all done based on the features stored in the corresponding database.

The first objective eases the modelling of both the geometric recognition of the object and the relationship among its components. The recognition process in this

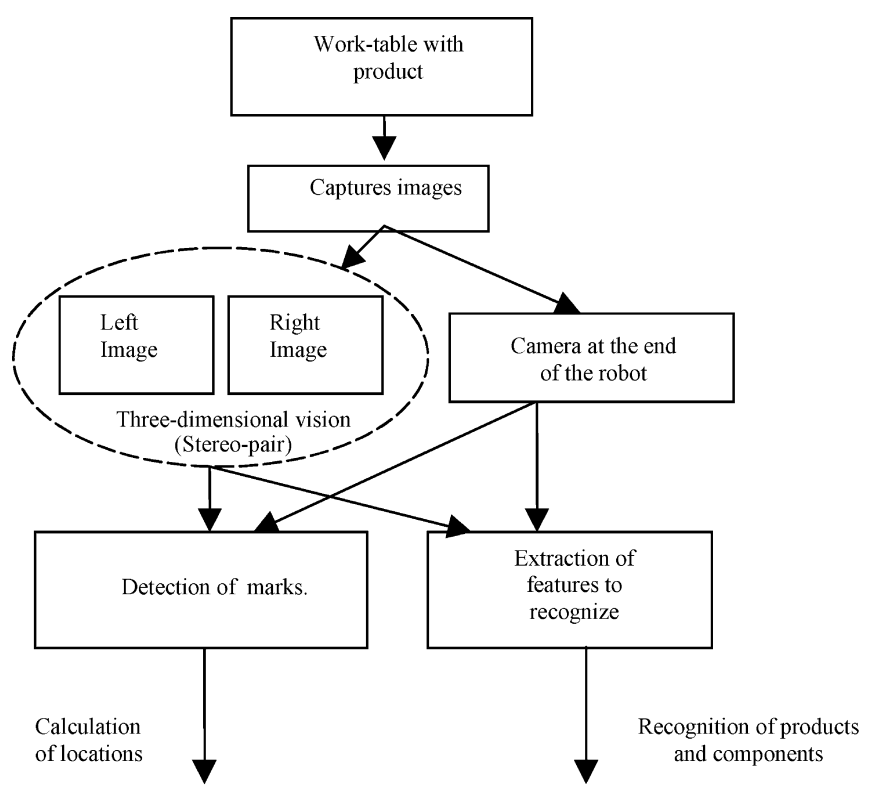

Fig. 2 Recognition and location process

case consists of defining and assigning a given model from which a product is to be disassembled, based on the minimum information (geometric and relationship) stored in the database.

Once the product to be disassembled has been modelled, having identified the precise components of the products, the second objective is to detect which component is to be disassembled. To do this, the images of the product captured are processed to obtain a series of characteristics that must be compared to the information stored in the database [4]. In Fig. 4a, the recognition process is described.

\subsubsection{Location}

Using a stereoscopic system, the manipulation process is carried out with a certain degree of autonomy, allowing
Fig. 3 a Stages of the recognition process and b Stages of the location process
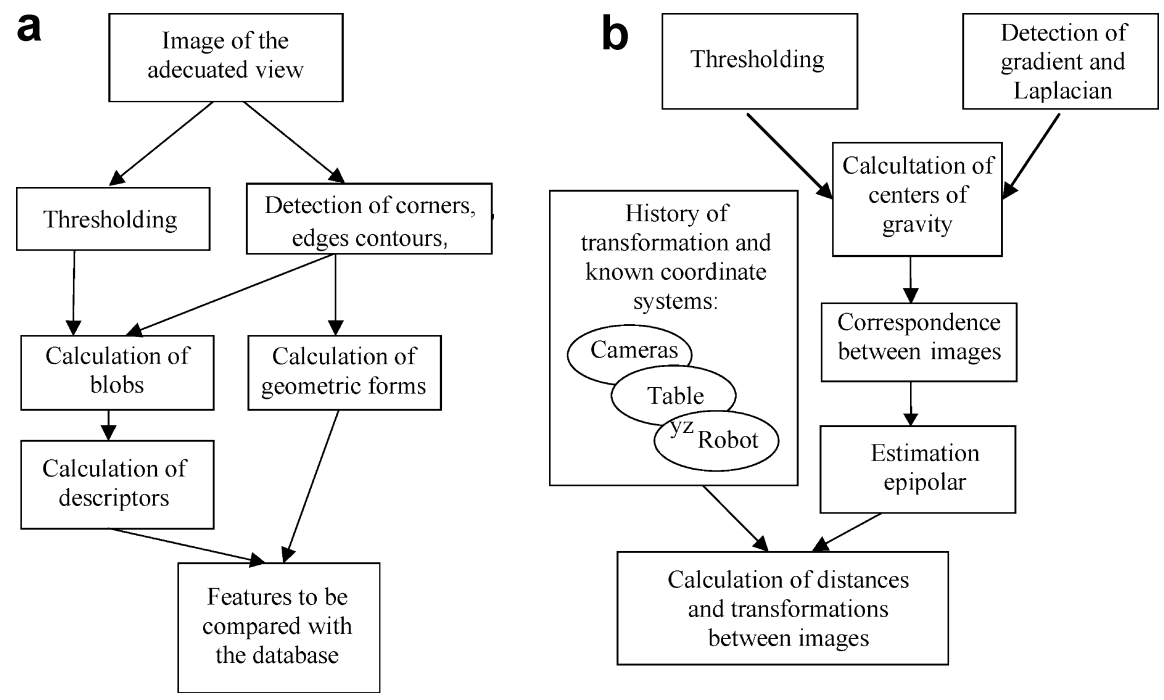
Fig. 4 Architecture and reference systems used

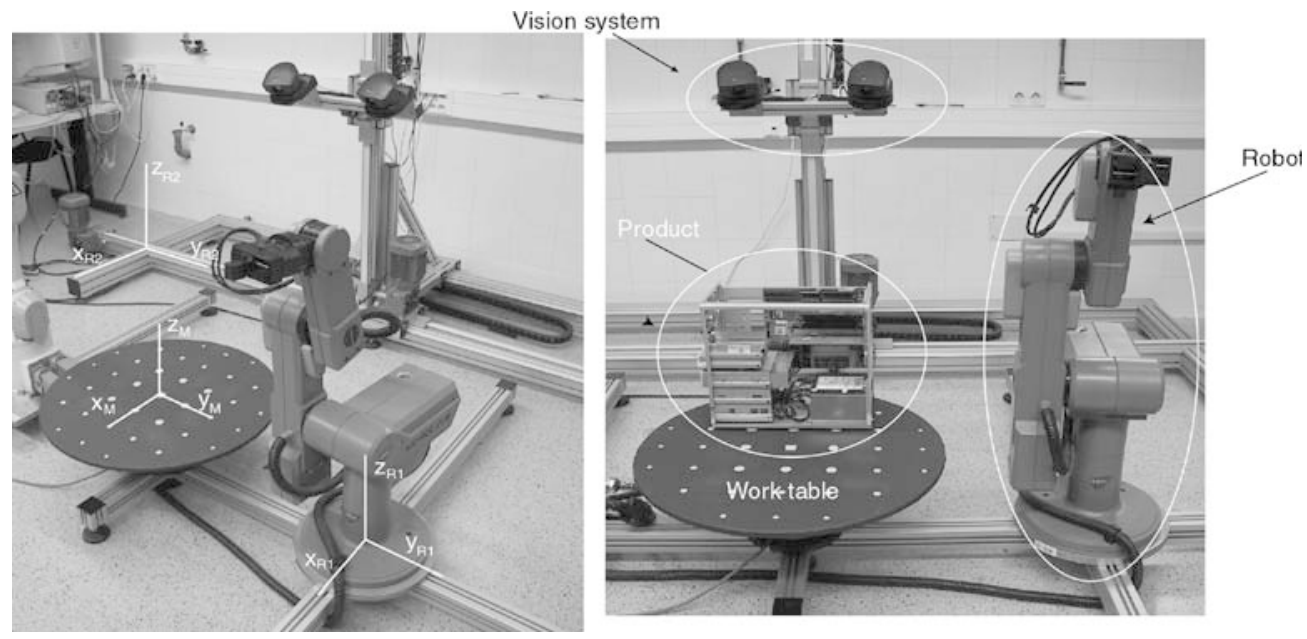

the system to work in a semi-automatic way, trying to avoid the need for any human operator as far as possible. At the same time, it contributes to greater flexibility so that it does not matter whether the manipulation process is dynamic.

Two problems arise in developing a location system based on computer vision. Firstly, the optical architecture of the camera (intrinsic parameters) is subject to the internal parameters (focal, distortions, centre points, etc.). Secondly, it is necessary to know the different coordinate systems of the elements that make up the disassembly cell, as well as the changes and variations that some of them experience with respect to others when some type of movement has taken place.

The intrinsic parameters that model the optics (lens and motorisation) can be obtained from the technical information provided by the manufacturer as well as from calibration techniques that allow the determination of the exact values of those parameters whenever the optics are modified.

Two cameras with motorised zoom lenses and a system of automatic focusing form the location system. The main problem with using video cameras whose lens parameters are not fixed is the necessity to recalibrate the system whenever changes in the optics take place. This occurs when it is necessary to acquire general images of the object in the first place and images of the details (use of the zoom lens) later on. In order to avoid the recalibration of the camera whenever there is a change in the position of the motor on which a lens is mounted (for example due to an automatic focus), a methodology based on providing a model of the lenses of the cameras has been used $[5,6]$.

To construct the model, it is necessary to know the maximum and minimum positions of the movement of the motors and to repeat the calibration process for each position of the steps of the motor.

The camera is calibrated for each position of the steps of the motor, and from the internal parameters obtained for each one of these positions, the function that interpolates and approximates the model of the behaviour of the camera, is computed. From this model, a focal value, a zoom lens, a distortion and a centre point are approximated and computed according to the position or motor step used.

From these estimations it is possible to obtain threedimensional models that relate the positions of the focus and zoom motor, which are introduced into the camera to move the lenses, with the value of an internal parameter that is obtained for each combination of focus and zoom lens. In this way, three main functions are achieved with which each of the different values obtained from the internal parameters of the camera (focal, distortion and centre point) are adjusted, by means of an interpolation process.

Once the internal parameters of the camera have been modelled according to the focus and zoom motor positions, the modelled intrinsic parameters of the camera (focus, centre point and distortions of lens) can be computed depending on the motor positions that have been used for the lenses. Given a determined position of the lens in each camera of the stereoscopic system, the value for each given parameter is computed.

The second problem is to obtain the extrinsic parameters of the cameras in the stereoscopic system This is the set of transformations (rotations and transfers) of the stereoscopic system that the cameras undergo when they make movements to adapt to a suitable direction and position to obtain a point of view allowing the acquisition of images.

The knowledge of this set of transformations allows the position and direction of each of the cameras in the stereoscopic system with respect to the object that is to be disassembled. Knowing the transformations that are taking place in the stereoscopic system is not really sufficient with regard to a component of that object to be established. Therefore, whenever movement is made to obtain a suitable point of view, it is also necessary to model the elements included in the disassembly cell.

This process basically consists of fixing a set of system co-ordinates for each one of the elements (manipulator robot, worktable and $y-z$ robot on which 
the stereoscopic system is located). The system of co-ordinates of the manipulator robot is taken as an absolute system of reference. In this way, the positions and directions are computed with respect to this system (Fig. 4). Once the reference systems, and where they are located are known, it is only necessary to know the variations that are taking place in them when each of the elements that make up the disassembly cell are moved.

The variations of the manipulator robot are its kinematics, i.e. the turns in the worktable are known and the change of location of the stereoscopic system is the position that the $\mathrm{y}-\mathrm{z}$ robot assumes.

Whenever a variation in some of the reference systems takes place it is stored temporarily, so that it is possible to know the transformation that has been produced.

From the images that are captured by the cameras in the stereoscopic system, a reconstruction process is made, which obtains the spatial co-ordinates of an object in the space. To compute these, a set of characteristics is extracted. Fundamentally, these are corners and segments of the edges of the objects that appear in the images, from which a process of correspondence between the characteristics obtained in both images is made, and subsequently, the epi-polar geometry that relates both views is computed [7,8].

The space co-ordinates (3D) of the product and components can be calculated once the intrinsic parameters of the cameras and the estimate of the epipolar geometry between the views obtained from the captured images are known, as well as the transformations that take place between table and cameras, which have been stored. The generic process used for the location is outlined in Fig. $3 b$.

\subsection{Modelling}

Before the disassembly process, it is necessary to have a model that provides sufficient information to the system for it to be able to do the disassembly.

The assemblies, as well as the products, are groups of components and/or sub-assemblies linked in them. There are different schemes representing them according to what they need to reflect. There are schemes that try to show a product from the designer's point of view, others from the user's point of view, and others based on the relationships among the different components or the characteristics of such components, etc. Therefore, when selecting the representation of a product to be disassembled, it is necessary to carry out a study of the characteristics that need to be reflected in the scheme, the type of disassembly to be done, and to whom such representation is directed.

The characteristics of the product that are to be reflected influence the representation through the granularity of the model of each of the components of which it is composed. For example, to disassemble a PC, the components to consider in this case are the cards, power supply, hard drive, etc., in the representation, it is not necessary to save information about the chips or other components of a card or any other element. With this representation it will not be possible to disassemble the chips or all those elements that are not represented, but if that disassembly level is not required, the scheme is correct and simpler.

In the literature, there are several ways of representing a product [9], using the complete knowledge of their components $[10,11]$, using the geometric characteristics of the products and components [12], by means of a graph constructed using geometric, topological and functional data of the product [13], using a componentsconnections graph to represent the product and the subsequent transformation into a tree [14, 15, 16], by means of the relationship among groups of components, using clusters [17] with directed graphs, considering the nodes as components and the edges as the relationships among them [18] (using "and/or graphs" [19, 20, 21, 22]).

In this study two models are used:

1. A relational model and its function to represent the relationships among the components through the existing contacts among them, which define new components (assemblies), so that a product can be expressed as the union of a set of assemblies with hierarchic relations through a graph [23]. This method has advantages over others, such as an easier and intuitive representation to allow the increase of the degree of granularity, from an existing representation, as well as to obtain the disassembly sequence, applying a set of pre-defined rules, and reducing it, in this case, to a search to determine what assemblies a component belongs to.

2. The geometric model is a multi-dimensional representation of the product, from polyhedral models of the components [24]. The geometric modelling must reflect the main physical characteristics of the components of the product (size, geometry, etc.) as well as the relationships among the different components of the product (contact surfaces, type of unions, locations of components, etc.). The model employed for the components (this information is stored in the components database) and the assembly model, which is generated automatically from the relational model and from the information processed by the computer vision system, are chosen (Fig. 5).

\section{Non-destructive partial disassembly of a PC}

\subsection{Disassembly cell}

A SCORBOT ER-IX robot with five degrees of freedom forms the cell used for the disassembly, with parallel fingers as a tool. A worktable is employed, which allows the orientation of the product, affording the system an additional degree of freedom. Finally, the system is completed with a stereoscopic system of cameras mounted on a Cartesian $\mathrm{y}-\mathrm{z}$ robot, whose purpose is to 
Fig. 5 Geometric model of table-top PC
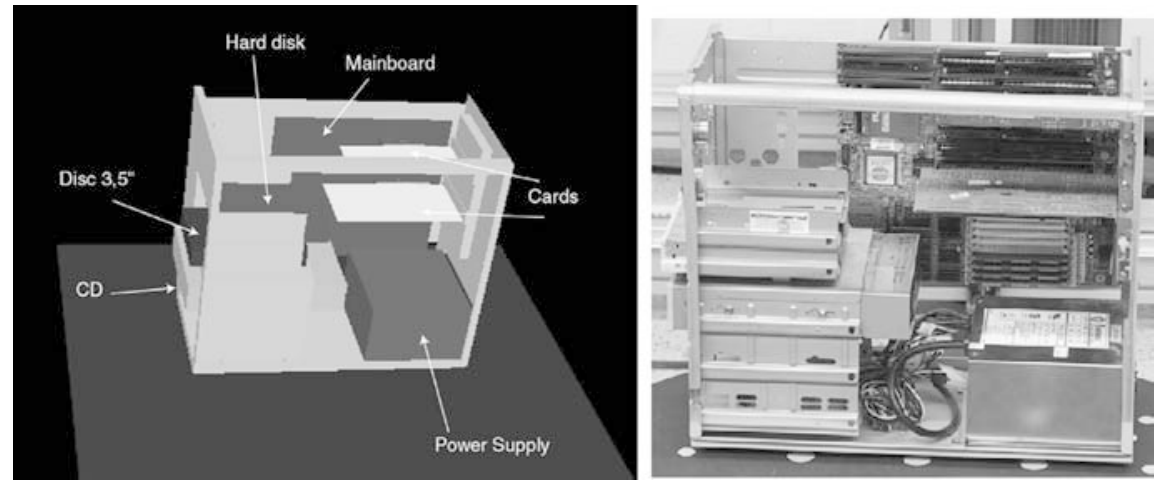

recognise and locate the components of the product (Fig. 4). Due to the limited robot workspace, manual displacements of the product can be made on the worktable. Such displacements are carried out manually since they cannot be carried out automatically on the table. As an example of the system's operations, the video card of the medium-sized upright PC that appears in Fig. 6 will be disassembled.

\subsection{Disassembly of the PC}

Once the PC has been positioned on the worktable, it is first necessary to recognise the type of product. In this case, the vision system captures different views of the product, using the table to orient it. In Fig. 6, the set of captured images made up of four views from each camera, which correspond to different views of the product, is shown. When the system has recognised the product (from the generated characteristics) as a medium-sized upright PC, the disassembly system selects the generic relational model of the PC product. With this relational model, the trajectory planner seeks the sequence of actions required to disassemble the video card from a graph.

A PC product is made up of a group of components (video card, memory card, hard drive, etc.) and/or subassemblies interconnected to them. A sub-assembly is composed of a group of interconnected components and/or sub-assemblies. In both cases the representations are made using a graph. There are different types of relationships among the components and the subassemblies. Each of these situations corresponds to a specific disassembly operation (e.g. remove "video card"), which we shall call an action.

It is possible to compute the correct disassembly sequence of the component's product "video card" by carrying out a sequence of actions. These actions are imposed by the graph of the PC product. The sequence of actions is obtained crossing the graph of PC product from the node that represents the component to be disassembled, "video card", until sub-assembling, which represents the final state of the PC product.

Considering the sequence of images in Fig. 6, it is necessary to remove the metal box cover from the PC, so the generator of movements calculates the trajectory for its disassembly.

Once the PC's metal box cover has been removed, the global planner uses the computer vision system and the knowledge base to complete the relational and geometric models of the product with the greatest possible detail from the information available. Next, frontal images of the product are captured with the purpose of recognising the cards and accessories that compose it (Fig. 7).

After the existing components of the product have been located, new relational and geometric models are generated. These include the recognised components with their precise locations and also indicate the removed components that have already been disassembled.
Fig. 6 Images captured by the left and right cameras of the stereoscopic system
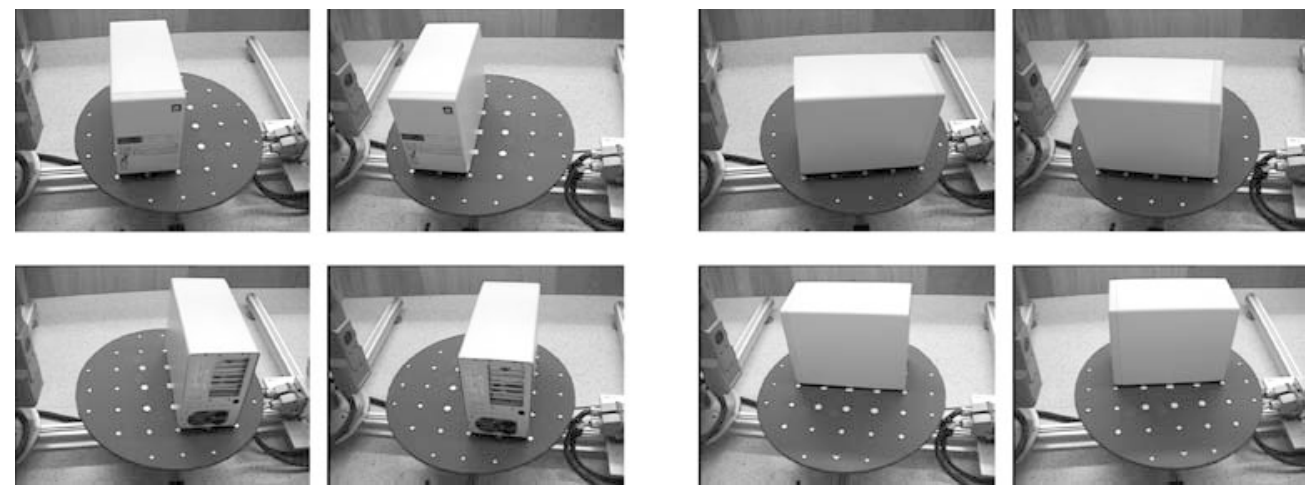
Fig. 7 Vision system acquire information. Example of video card and CDROM
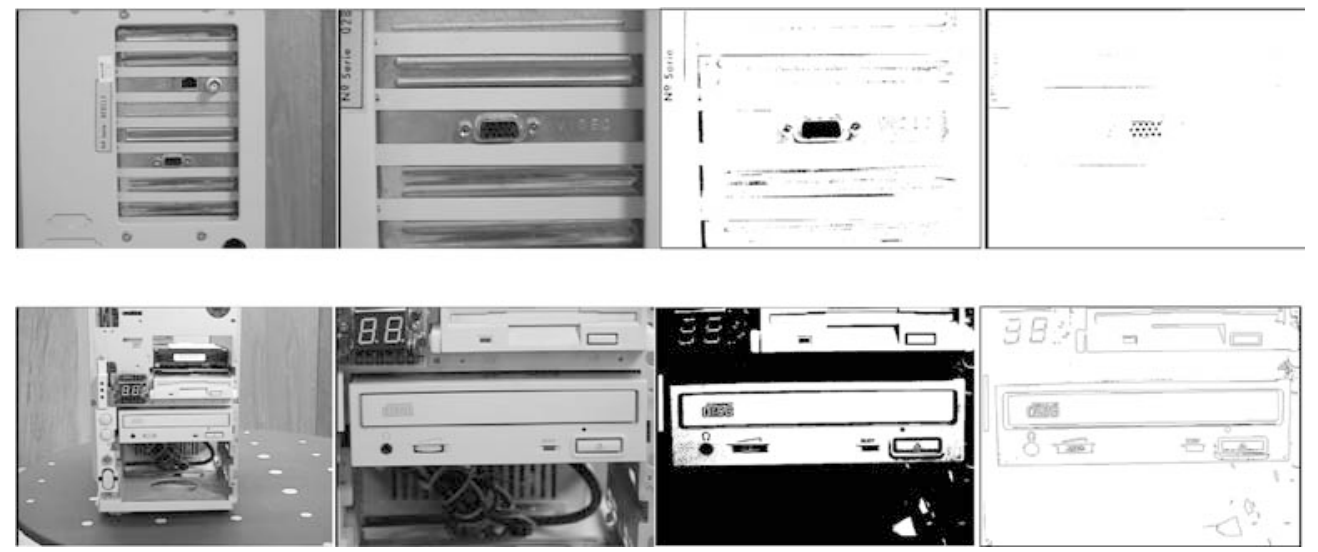

In Fig. 5, the geometric model generated at this moment is shown. With this new product modelling, the sequence planner determines the operations for disassembling the component "video card".

Finally, once the generator has established the movements to be made in the trajectories, the simulation of the disassembly process for the video card (Fig. 8) is realised and the real disassembly is carried out according to this simulation (Fig. 9).

\section{Conclusions}

In this article, a study aimed at obtaining a nondestructive automatic disassembly system for personal computers has been presented. To do this, the different components that integrate the disassembly system and the relationships among them have been described. Following this description, the different aspects to be
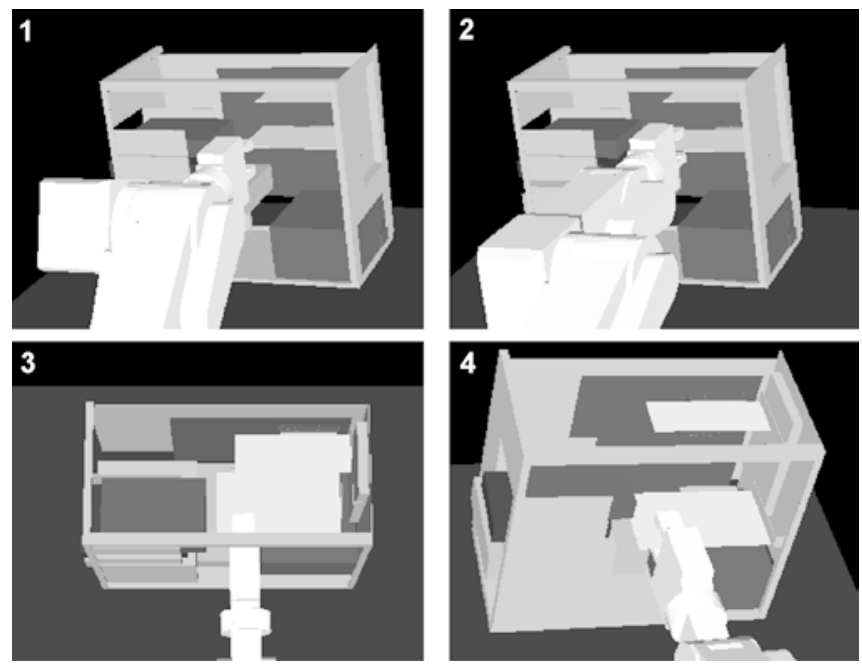

Fig. 8 Photograms of simulation sequence for disassembly trajectory of the component "video card" from different view points
Fig. 9 Sequence of real photograms for the disassembly of the component "video card"
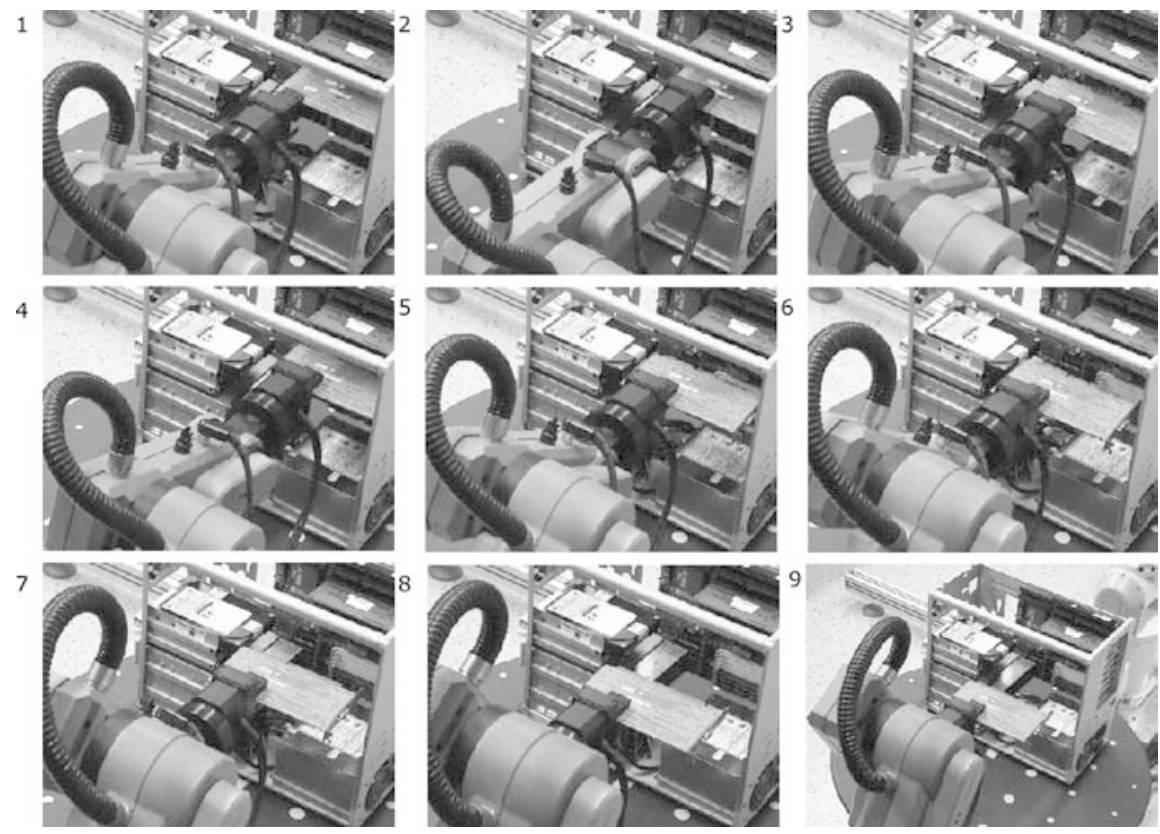
considered for the disassembly of each element in the system and the details of the processes have been shown.

The main sensory component of the disassembly system is composed of a computer vision system made up of stereo cameras mounted on a y-z robot. This optical system captured the information required to carry out the two phases described in this article: i.e. recognition and location.

As shown in recent research in the field of automatic disassembly, it is necessary to have a representation of the product that permits the disassembly of the target components. A representation of the product, based on two models, has also been proposed: i.e. relational and geometric. The first one provides a hierarchic vision of the unions between the different components of the product, whereas the second one offers multi-dimensional information of the product. Both models included in the disassembly system provide, a priori, the information required for the entire process.

The results obtained from the disassembly of a previously modelled PC are shown. The tests demonstrate the good operation of the proposed process, thanks to the flexibility of the computer vision system, which allows the disassembly system to function correctly in spite of the deficiencies inherent in the model and/or the correspondence between the model and the real product to be disassembled.

We are currently working on providing greater robustness to the sensing system by integrating force control as well as including alternative distance sensors, such as lasers. We are also testing the use of a second robot for the supervision of the disassembly process.

Acknowledgements This work was funded by the following Spanish CICYT project "Sistema Robotizado de Desensamblado Automático basado en Modelos y Visión Artificial" (TAP1999-0436).

\section{References}

1. Hesselbach J, Kühn M (1998) Survey of processes at disassembly companies. Proceedings, IFAC Workshop of Intelligent Assembly and Disassembly (IAD'98), Bled, Slovenia, pp 99103

2. Gungor A, Gupta SM (1999) Issues in environmentally conscious manufacturing and product recovery: a survey. Comput Indust Eng 36:811-853

3. Gil P, Puente ST, Torres F, Pomares J, Candelas FA (2001) Data fusion from multiple cameras for automatic disassembly. Proceedings, IFAC Workshop of Intelligent Assembly and Disassembly (IAD'01), Canela, Brazil

4. Najjari H, Steiner SJ (1996) 3D CAD-based object recognition for a flexible assembly cell. In: Intelligent Robots and
Computer Vision XV: Algorithms, Techniques, Active Vision, and Materials Handling 2904:167-177

5. Willson RG (1994) Modeling and calibration automated zoom lenses. Phd. Thesis Department of Electrical and Computer Engineering, Carnegie Mellon University

6. Ahmed Moumem, Farag AA (2000) A neural optimization framework for zoom-lens camera calibration. Proceedings of IEEE, International Conference on Computer Vision (CVPR'2000), Hilton Head Island, SC, 1:403-409

7. Faugeras O (1993) Three dimensional computer vision. A geometric viewpoint, Cambridge

8. Hartley and Zisserman (2001) Multiple view geometry in computer vision. Cambridge

9. O'Shea B, Grewal SS, Kaebernick H (1998) State of the art literature survey on disassembly planning. J Concurr Engin Res Appl 6(4):345-357

10. Subramani AK, Dewhurst P (1991) Automatic generation of product disassembly sequences. Annals of the CIRP 40(1)

11. Yokota K, Brough DR (1992) Assembly/disassembly sequence planning. Assembly Autom 2(3):31-38

12. Qian WH, Pagello E (1994) On the scenario and heuristic's of disassemblies. Proceedings, IEEE International Conference on Robotics and Automation, pp 264-271

13. Zussman E, Lenz E, Shpitalni M (1990) An approach to the automatic assembly planning problem. Annals of the CIRP 39(1)

14. Kuo TC (2000) Disassembly sequence and cost analysis for electromechanical products. Rob Comput Integr Manuf 16:43-54

15. Zhang HC, Kuo TC (1996) A graph-based approach to disassembly model for end-of-life product recycling. Proceedings, IEEE/CPMT Electronics Manufacturing Technology Symposium, pp 247-254

16. Zhang HC, Kuo TC (1997) A graph-based disassembly sequence planning for EOL product recycling. Proceedings IEEE/CPMT Electronics Manufacturing Technology Symposium, pp 140-151

17. O'Seha B, Kaebernick H, Grewal SS (2000) Using a cluster graph representation of products for application in the disassembly planning process. Concurr Eng Res Appl 8(3):158-170

18. Puente ST, Torres F, Gil P (2001) An approach to disassembly sequence generation. Proceedings IFAC Workshop of Intelligent Assembly and Disassembly (IAD'01), Canela, Brazil

19. De Mello HLS, Sanderson AC (1995) AND/OR graph representation of assembly plans. IEEE Transaction On Robotics And Automation 6(2):188-199

20. Lee S, Yi C (1993) Forced-based reasoning in assembly planning. Vision Sensors and Control for Automated Manufacturing Systems, SPIE 2063:97-108

21. Perlewitz H, Müller K, Seliger G (1998) Usage mode and effects analysis to support disassembly process planning. Proceedings, IFAC Workshop of Intelligent Assembly and Disassembly (IAD'98), Bled, Slovenia, pp 11-16

22. Zorc S (1998) Learning in assembly planning. Proceedings, IFAC Workshop of Intelligent Assembly and Disassembly (IAD'98), Bled, Slovenia, pp 17-22

23. Puente ST, Torres F, Pomares J (2001) Product disassembly scheduling using graph models. Proceedings of SPIE, International Symposium on Intelligent Systems and Advanced Manufacturing, Newton, USA, 4569:63-70

24. Pomares J, Torres F, Puente ST (2001) Disassembly movements for geometrical objects through heuristic methods. Proceedings of SPIE, International Symposium on Intelligent Systems and Advanced Manufacturing, Newton, USA, 4569:71-80 